\title{
Transatlantica
}

Revue d'études américaines. American Studies Journal

\section{«I Speak According to the Book » : écriture et logos dans Nixon in China de John Adams et Alice Goodman}

Mathieu Duplay

\section{OpenEdition}

Journals

Édition électronique

URL : https://journals.openedition.org/transatlantica/6395

DOI : 10.4000/transatlantica.6395

ISSN : $1765-2766$

Éditeur

Association française d'Etudes Américaines (AFEA)

Référence électronique

Mathieu Duplay, " «I Speak According to the Book » : écriture et logos dans Nixon in China de John Adams et Alice Goodman », Transatlantica [En ligne], 1 | 2013, mis en ligne le 16 décembre 2013, consulté le 01 février 2023. URL : http://journals.openedition.org/transatlantica/6395 ; DOI : https:// doi.org/10.4000/transatlantica.6395

Ce document a été généré automatiquement le 1 février 2023.

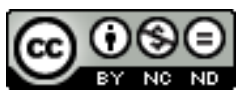

Creative Commons - Attribution - Pas d'Utilisation Commerciale - Pas de Modification 4.0 International - CC BY-NC-ND 4.0

https://creativecommons.org/licenses/by-nc-nd/4.0/ 


\title{
"I Speak According to the Book» : écriture et logos dans Nixon in China de John Adams et Alice Goodman
}

\author{
Mathieu Duplay
}

1 Bien que consacré à des faits réels - la visite à Pékin du président Nixon en février 1972, prélude à la reprise des échanges diplomatiques entre les États-Unis et la Chine communiste - Nixon in China (1987) de John Adams et Alice Goodman ne se laisse qu'en partie appréhender comme un opéra historique. L'événement est dépeint avec un grand souci d'exactitude (Goodman, 1987b, 11-12); toutefois, l'enjeu consiste moins à évoquer un passé encore récent et pourtant déjà bien éloigné des réalités

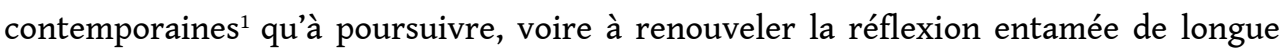
date en Amérique sur les enseignements dont est porteuse la rencontre avec la civilisation chinoise.

2 Vue depuis les États-Unis, la Chine avait rarement semblé aussi mystérieuse qu'au début des années soixante-dix, après vingt-cinq ans d'isolement diplomatique et à l'issue de la Révolution culturelle, source de stupéfaction pour la plupart des observateurs extérieurs (Angeloff, 39) ; aussi le voyage de Nixon permet-il à Adams et à Goodman de souligner l'étrangeté d'un lieu traditionnellement perçu comme l'ailleurs par excellence. Pourtant, on chercherait en vain la moindre trace d'essentialisme esthétisant dans un ouvrage où la découverte des mondes lointains apparaît avant tout comme un enjeu éthique. Comme l'écrit Véronique Béghain, le spectacle du dissemblable auquel convie Nixon in China permet surtout de distinguer des ressemblances; l'attention se fixe sur une expérience du même au sein du différent (Béghain, 29). Jamais la distance entre les deux cultures n'est minimisée au point de faire perdre de vue les spécificités de la civilisation chinoise, mais cet écart persistant n'autorise pas à tenir la Chine pour une figure de l'altérité radicale, enjeu possible d'une entreprise d'appropriation néo-coloniale. Au contraire : l'épreuve de l'étranger donne lieu à un questionnement identitaire dont nul n'est exempté, à commencer par les voyageurs eux-mêmes; le détour par l'Asie fait, par comparaison, surgir l'inconnu 
au cœur du familier lorsque les Américains ont la surprise de retrouver, si loin de chez eux, un peu de leur quotidien : "I saw / A snow moon on our way here. Snow! / Snow over China! Think of that! » s'exclame Mrs. Nixon peu après son arrivée (Goodman, 1987a, 31). Nouvelles ou nouvellement découvertes, ces réalités insoupçonnées acquièrent bien vite une valeur d'exemplarité : parcourir la Chine, c'est aussi apprendre à se voir soimême, non pas tel que l'on est, mais tel que l'on pourrait enfin devenir pour peu que la routine ne porte pas atteinte à la lucidité tout juste acquise, intuition de la magie cachée au plus intime des êtres. Aussi le voyage à Pékin se présente-t-il in fine comme une étape du dialogue que l'Amérique entretient avec elle-même, non qu'elle se montre indifférente à ce qui n'est pas elle, mais au contraire parce que la distance permet de substituer, à la fausse évidence de la présence à soi, la conscience aiguë de l'intervalle et de l'écart au cœur du même.

[L]et us, in these next five days

Start a long march on new highways

In different lanes, but parallel

And heading for a single goal,

proclame Nixon au cours de la réception officielle qui clôt l'acte I (36). S'il est vrai que les parallèles ne se rencontrent qu'à l'infini, alors la destination vers laquelle il se propose de cheminer ne saurait figurer sur aucune carte, trait qu'elle partage avec l'utopie. C'est en tout cas ce que suggère la métaphore de l'autoroute: d'emploi idiomatique aux États-Unis, le vocable "highway» acquiert une résonance inattendue dans ce nouveau contexte; associé à la geste maoïste et au souvenir de la Longue Marche, il incite à s'interroger sur ce que pourrait devenir la Grand-Route, topos de la culture américaine, si d'aventure elle se prolongeait vers l'ouest, par-delà l'Océan Pacifique et jusqu'aux confins de la Mandchourie.

Lorsque les auteurs de Nixon in China évoquent l'Extrême-Orient afin d'élaborer une nouvelle poétique américaine au contact de la civilisation chinoise, ils s'inscrivent de plus ou moins bon gré dans une double filiation à la fois littéraire et musicale. De ce point de vue, le livret d'Alice Goodman prend place dans une longue tradition que domine la figure d'Ezra Pound ; quant à la musique post-minimaliste de John Adams, elle doit beaucoup, de son propre aveu, aux leçons de John Cage, adepte du Yi King et féru de philosophie taoïste.

5 Avec ce riche passé, la librettiste et le compositeur entretiennent des relations ambivalentes. Adams n'a pas de mots assez forts pour souligner combien l'apport théorique de John Cage lui paraît essentiel, ni à quel point la publication de Silence (1968) a transformé la musique américaine: «I read this book as a 20-year-old aspiring composer and found what Cage had to say [...] the musical equivalent of the young Martin Luther's nailing his theses to the door of the Wittenberg church » (Adams, 2010). Toutefois, si Adams fait l'éloge du penseur, c'est pour aussitôt critiquer le compositeur, dont il juge les œuvres au plus haut point rébarbatives :

I think much of [Cage's] later work is fundamentally, even tediously, didactic. A work like « ' '33" « is a demonstration, a lesson in how to listen, so to speak. But to equate its artistic value, as some have, with a work like " The Rite of Spring » is to confuse art with philosophy.

(« Up Front : John Adams », 2010)

6 Quant à l'écriture de Goodman, elle doit plus, sur le plan formel, à l'exemple des poètes métaphysiques anglais qu'à celui des Cantos. Tout entier rédigé en tétramètres iambiques, le vers préféré d'Andrew Marvell, le livret de Nixon in China tend davantage 
vers une sorte de néo-classicisme que vers une pratique de la juxtaposition et de l'hétérogène inspirée de l'esthétique poundienne; Alex Ross a même proposé un rapprochement (suggestif mais discutable) entre l'aria de Pat Nixon (Goodman, 1987a, 41-43) et « The Emperor of Ice Cream » de Wallace Stevens, auteur que Marjorie Perloff tient pour le plus brillant représentant d'une poétique moderniste en tous points opposée à celle de Pound (Perloff, 1-32). On n'en constate pas moins que Goodman se montre tout aussi sensible que Pound à l'historicité de la littérature ; comme lui, elle témoigne d'une préférence marquée pour la poésie des époques antérieures au romantisme : " the Pound Era is the era when the norms of the Romantic crisis poem [...] were exploded, when poetry found that it could once again incorporate the seemingly alien discourses of prose without losing its identity " (Perloff, 22). Cette découverte, Goodman la confirme à sa manière lorsqu'elle récrit en vers les minutes des entretiens entre Nixon et Mao ou façonne un poème dramatique en trois actes à partir de documents d'archives. De Cage à Adams, de Pound à Goodman, pas de transmission continue, nul passage de témoin, mais la persistance d'un questionnement dont le propos d'Adams suggère bien les affinités avec la philosophie.

7 Or l'enjeu immédiat de cette réflexion, chez Pound comme chez Cage, n'est autre que l'écriture entendue au sens premier d'inscription, de marque lisible, sujette à des déterminations différentes d'un système sémiotique à l'autre. Si Pound accorde une telle importance à la pensée et à la littérature chinoises, c'est, dit-il, parce que le recours aux idéogrammes les protège contre la tentation de l'abstraction à outrance et les dissuade de céder à la fascination de l'incompréhensible. "Chinese ideogram does not try to be the picture of a sound, or to be a written sound recalling a sound, but it is still the picture of a thing; [...] [i]t means the thing or the action or situation 》 (Pound, 21). Aussi l'Europe serait-elle bien inspirée, selon lui, de suivre l'exemple chinois, puisque le choix de l'écriture alphabétique l'a privée de semblables garde-fous (19). Que les spéculations de Pound soient suspectes aux yeux des sinologues n'enlève rien à l'essentiel. D'une part, soutient-il, une technique d'écriture peut infléchir le rapport qu'une civilisation donnée entretient avec le langage et avec le monde ; d'autre part, cette culture ne peut aisément en prendre conscience par elle-même : elle a besoin pour cela de se comparer à une tradition exogène dont elle ne partage pas les choix. Sur ce point, Cage développe une argumentation analogue. En Occident, composer de la musique, c'est d'abord l'écrire; le compositeur n'opère pas à l'aide de sons, ni même d'images mentales en attente de réalisation sonore, mais trace des notes sur du papier. C'est pourquoi, selon lui, la réflexion contemporaine sur les techniques d'écriture préfigure à coup sûr un renouvellement imminent de la création musicale :

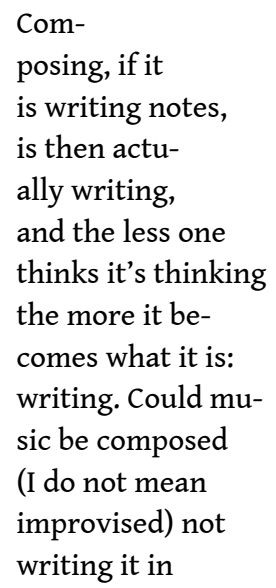




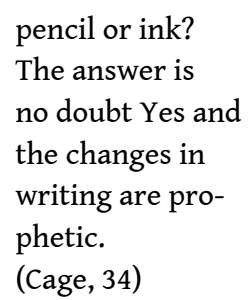

Pour ces deux auteurs, l'écriture n'a pas nécessairement pour utilité de noter les sons ceux de la langue ou de la composition musicale, peu importe au fond; pour sa part, Cage doute même qu'il y ait un sens à s'interroger sur les rapports entre la graphie et les phénomènes sonores censés à tort s'y rattacher : « Composing's one thing, performing's another, listening's a third. What can they have to do with one another?» (15) Aussi s'interrogent-ils sur la véritable fonction de l'écrit, qui est de guider le déploiement de la pensée en favorisant d'emblée certains modes d'activité cognitive aux dépens d'autres qui du coup se trouvent négligés, voire discrédités. Récurrente tout au long du $20^{\text {ème }}$ siècle nord-américain, cette problématique trouve sans doute sa formulation la plus développée dans les travaux de Marshall McLuhan, qui à l'instar de Pound oppose l'écriture alphabétique à la " méthode idéogrammatique » chère à la tradition chinoise et à qui Cage se réfère fréquemment à partir des années soixante-dix. Selon McLuhan,

[...] « linear thinking » is not merely a figure of speech, but a mode of activity which is peculiar to the anterior regions of the left hemisphere of the brain. [...] [T] he use of the alphabet, with its emphasis on linear sequence, stimulates mastery of this area of the brain in cultural patterns. [...] [T] he written alphabet, with its linear structure, was able to create the conditions conducive to the development of Western science, technology, and rationality.

(McLuhan, 1989, 58)

Telle est la problématique qu'Adams et Goodman héritent de leurs prédécesseurs et que, comme eux, ils tentent d'explorer à la lumière de l'exemple chinois. Tenants d'une pensée politique conservatrice, défenseurs des valeurs traditionnelles, les époux Nixon pressentent pourtant dès leur arrivée en Chine l'imminence d'un bouleversement spirituel, véritable révolution intérieure qui les fera accéder à un nouvel état de conscience ; sans le savoir, Mrs. Nixon fait écho à Cage lorsqu'elle annonce les surprises qui attendent une Amérique plongée dans un sommeil plein de rêves prophétiques :

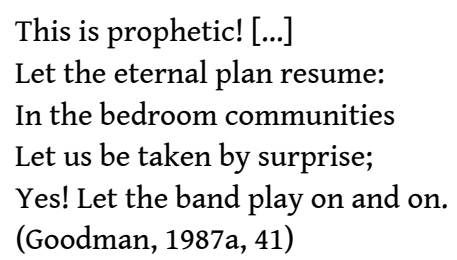

10 S'il en est ainsi, c'est que le propre de l'événement qu'ils vivent est de les confronter à de nouveaux modes de fonctionnement sémiotique à mesure qu'ils explorent le pays du Petit Livre rouge et des Analectes de Confucius, textes que tout oppose en termes idéologiques mais qui ont en commun d'illustrer les pouvoirs insoupçonnés de l'idéogramme. Nixon in China œuvre ainsi à l'élaboration d'une nouvelle " grammaire " attentive à la manière dont les procédés d'écriture structurent l'expérience et le devenir des êtres (McLuhan, 1989, 33) ; c'est sa manière de contribuer à la réinvention de soi qu'appelle le voyage en Chine, premier contact avec un ailleurs où des modalités non alphabétiques de l'inscription interrogent les catégories habituelles de l'esthétique et de l'épistémologie. 
11 Indéniablement doté d'une pertinence philosophique, ce travail ne relève pourtant pas de la philosophie à proprement parler, comme Adams le laisse entendre lorsqu'il critique le "didactisme" de Cage. Nixon in China pousse jusqu'à son terme un questionnement dont il ressort que l'écriture n'est pas un objet de pensée car elle se déploie à distance du logos dans une dimension de l'expérience concrète qui ne se donne pas d'emblée la connaissance pour horizon : éprouver le mystère de l'écrit dans son opacité sensible, et non chercher à l'aborder de quelque manière que ce soit à l'aune d'une idéalité à laquelle il demeure une fois pour toutes étranger, tel est ici l'enjeu. À moins bien sûr qu'il ne faille entendre par " philosophie » autre chose que ce que l'on désigne ainsi en Occident et aller jusqu'au bout d'une démarche qui incite à rechercher dans la tradition chinoise, par exemple chez Laozi (Lao-tseu), une réponse non logocentrique à la question « Qu'appelle-t-on penser?».

Winding and twisting: she cannot be named;

She reverts back to when there was beingless.

She is called « the shape without a shape "; " the image of what is not a thing. "

She is obscure light!

(Laozi, 31)

12 Afin d'approfondir cette problématique, on examinera d'abord, dans les pages qui vont suivre, la comparaison que propose Nixon in China entre deux manières qu'a l'écriture de structurer le rapport au monde selon qu'elle est alphabétique ou idéogrammatique. Jusqu'à un certain point, cette analyse confirmera le bien-fondé d'un rapprochement avec la pensée de McLuhan, mais elle en fera également percevoir les limites. Étudier Nixon in China à la lumière de Understanding Media, c'est aussi s'interroger sur le média nouveau qu'inventent Adams et ses collaborateurs, sur ses rapports avec les médias existants, voire sur la fonction inattendue qu'il leur attribue dans un contexte transformé par son apparition. Or la principale innovation de Nixon in China consiste de ce point de vue à élaborer une dramaturgie du livre dont ne saurait rendre compte la grammatologie trop sommaire que propose McLuhan. On ne saurait, du reste, lui en faire grief, car la pensée de l'écriture vers laquelle fait signe Nixon in China n'existe nulle part en philosophie, pas même chez Derrida qui pourtant s'interroge sur ses conditions de possibilité, mais pour aussitôt l'exclure de son champ d'étude. Le présent travail n'aura pas pour ambition d'élaborer cette grammatologie nouvelle, mais tout au plus de suggérer, sur le mode hypothétique, à quoi elle pourrait ressembler.

\section{Du médium au média : écriture et partage du sensible}

13 Le modernisme littéraire a pour particularité de mettre en valeur l'autonomie du médium, avance Charles Bernstein (94); avant tout consciente d'être écrite, au sens matériel du terme, la poésie moderniste se reconnaît à ce qu'elle met en œuvre ce qu'écrire veut dire, commente Isabelle Alfandary (10). On peut souscrire sans réserve à cette affirmation tout en observant qu'elle sous-entend une prise de position sur l'indépendance du littéraire, de l'écriture appréhendée cette fois dans sa dimension esthétique et savourée pour son intransitivité, ainsi qu'Alfandary l'ajoute aussitôt (10) à la suite de nombreux auteurs modernistes qui trouvent en Mallarmé leur principal inspirateur. Or si Nixon in China prend acte - en écho à John Cage et, indirectement, à Ezra Pound - des interrogations modernistes sur l'écriture, force est de constater que la question posée par Adams et Goodman ne se laisse que malaisément formuler en ces termes. Ce qui est en jeu dans cet opéra, c'est au contraire l'inéluctable transitivité de 
l'écrit, son aptitude à exercer simultanément une influence dans tous les domaines de la pensée et de l'expérience y compris les plus éloignés de celui que l'on réserve d'ordinaire à la littérature; et si cette capacité s'avère aussi prononcée, c'est que la problématique de l'inscription ne se laisse pas circonscrire à la sphère linguistique mais engage l'ensemble de notre rapport au monde, comme en témoigne la manière dont Cage emploie le mot de "writing " pour désigner indifféremment le discours écrit et la notation musicale, qui peut se passer entièrement des mots.

14 À la question du médium défini comme le matériau scriptural dont se sert le poète, on est donc tenté de substituer celle du média au sens de McLuhan, agent d'un partage du sensible bien différent de celui qu'étudie Jacques Rancière. Au nombre des "prolongements de l'humain » ("extensions of man ») grâce auxquels nous sommes en mesure d'étendre notre système nerveux par-delà les frontières de notre corps, l'alphabet se montre à lui seul capable de déséquilibrer notre rapport sensoriel au monde en faveur du visuel et de tout ce qui s'y rattache, au premier chef une certaine conception de l'idéalité, de la causalité et du sujet pensés sur le modèle de l'activité de lecture. Selon McLuhan, ce qui fait les frais de cette transformation, c'est l'élément auditif-tactile (McLuhan, 1989, 59), avec lequel l'idéogramme n'a pas rompu car il agit à la manière d'une Gestalt indécomposable (McLuhan, 1964, 92); du reste, ajoute-t-il, l'essor des nouveaux médias audio-visuels et informatiques est en passe de corriger ce biais caractéristique de la culture occidentale (McLuhan, 1989, 88). De ce point de vue, la principale innovation de Cage consiste à inventer un rapport nouveau entre l'œil, l'oreille et la main : chez lui, la notation musicale ne propose plus une analyse du flux sonore selon les hauteurs et les durées, mais encourage une approche de type gestaltiste, par exemple dans les Song Books (1970). Peu importe en définitive que l'on mette ou non par écrit les sons musicaux, si tant est que cette alternative ait un sens; l'essentiel est de ménager une voie d'accès à la musique qui ne trahisse plus, d'emblée et par principe, sa nature auditive/haptique et qui lui permette enfin d'entrer dans un rapport non conflictuel avec la vision.

15 On mesure sans peine l'intérêt de cette réflexion pour un compositeur (Adams) et un écrivain (Goodman) attelés à un projet d'ouvrage qui mêle poésie, musique et représentation scénique, d'autant que cet opéra a pour sujet une rencontre au sommet minutieusement organisée en fonction des exigences de la retransmission télévisée. De fait, le livret de Nixon in China cherche à proposer une nouvelle répartition du sensible, même s'il ne va pas jusqu'à inventer pour cela des modes originaux d'inscription.

It's prime time in the U.S.A.

Yesterday night. They watch us now;

The three main networks' colors glow

Livid through drapes onto the lawn,

chante Nixon à sa descente d'avion, peu après sa poignée de main historique avec Chou En-lai ${ }^{2}$ (19). Goodman recourt ici au procédé traditionnel de l'hypotypose : dans ces quelques vers, la fonction première du logos est de donner à voir. Or ce qui est vu, c'est d'abord la vision en tant que telle, les transformations qu'un même contenu subit sous l'action de différents médias, et non ce contenu lui-même. Perçue par les téléspectateurs américains, la rencontre des deux hommes diffère subtilement de la scène qui vient de se dérouler devant le public de l'Opéra: lorsque j'assiste à une représentation de Nixon in China, je suis là maintenant («now»), alors que l'image télévisée, pourtant retransmise en direct, appartient au passé (" yesterday night») en raison du décalage horaire ${ }^{3}$. À la faveur de cette mise en abyme s'introduit un écart au 
sein du visible (Kamuf, 89), associé à un effet de brouillage puisque le texte ne décrit pas l'image télévisée mais se contente d'évoquer ses reflets à travers les rideaux tirés, autrement dit sa résonance visuelle, analogue aux échos planétaires qu'éveille l'événement. Le contour intelligible, les délimitations rigoureuses s'estompent au profit d'une forme de diffusion propice à des effets de synesthésie : d'un côté, l'œil cesse de privilégier l'écran du téléviseur et explore tout l'espace du visible, à la manière d'une écoute non directionnelle; de l'autre, la poignée de mains entre Nixon et le premier ministre chinois attire l'attention sur les liens qu'entretiennent la tactilité et l'haptique avec l'exercice du pouvoir politique dans une société où la transmission de l'information ne s'opère plus prioritairement par l'intermédiaire de la presse écrite. Paradoxalement, le recours à un procédé rhétorique censé privilégier la vision finit ainsi par produire l'effet inverse, sans doute parce que tout est en l'occurrence affaire de contexte: la situation n'en appelle pas au regard analytique d'un observateurlecteur détaché, mais à l'exploration intuitive et multi-sensorielle d'un environnement non euclidien où le temps ne s'écoule plus de manière linéaire, puisque l'on peut y être à la fois « aujourd'hui » et " hier ». C'est en ce sens, sans doute, que Nixon est bel et bien "en Chine", ce qui d'avance contribue à rendre problématique son allusion aux voies parallèles d'une autoroute à l'américaine, si semblables à des vers juxtaposés sur la page. Comme l'indique en effet McLuhan, l'espace des mathématiciens, celui où les parallèles se rejoignent à l'infini, se présente lui-même comme un produit de la culture visuelle à laquelle l'alphabet donne naissance, et il ne saurait être confondu avec l'espace réel, celui des astronomes ou des explorateurs. "There are no straight lines in space; as Einstein pointed out parallel lines do not meet in infinity. They simply curve back upon themselves» (McLuhan, 1989, 133).

Selon McLuhan, la tradition philosophique fondée par Platon et Aristote est pour l'essentiel d'inspiration dialectique, si par "dialectique » on entend la parole abordée sous l'angle de l'idéalité et considérée indépendamment du discours effectif; liée à la prédominance de l'alphabet phonétique qui permet à l'écrit de se donner pour la représentation silencieuse du discours, cette orientation s'est trouvée confirmée, depuis la Renaissance, par l'invention de l'imprimerie. De ce fait, la philosophie occidentale privilégie l'abstraction, "with its [...] emphasis on [...] absolutes, and on correct thought form (sequence), irrespective of content or audience " (McLuhan, 1989, 33). En revanche, elle peine à concevoir un rapport au monde fondé sur un mode d'appréhension autre que visuel, ce qui la rend inapte à rendre compte des mutations technologiques en cours (34). McLuhan propose donc de réhabiliter la grammaire, qu'il définit comme l'étude de la manière dont le logos structure le devenir ; dans cet esprit, il lui recommande de s'appuyer sur l'étymologie ainsi que sur une forme d'exégèse attentive à la pluralité des niveaux de signification ("multiple-level exegesis », 33).

Si Nixon in China présente une pertinence pour la philosophie, c'est notamment parce qu'elle y est soumise à un examen critique analogue à celui que préconise McLuhan, quand elle n'y est pas traitée, pour les raisons qu'il énumère, comme un objet de satire. "Ah, the philosopher! I see / Paris can spare you then", s'exclame Mao (Goodman, 1987a, 21) lorsqu'il fait la rencontre de Kissinger, homme politique mais aussi universitaire renommé. Manifestement ironique, ce commentaire fait mouche, car le personnage de Kissinger ne brille guère par son habileté dialectique : à l'acte I, il peine à suivre le raisonnement de Mao, certes particulièrement retors ; il réapparaît à l'acte II grimé en tortionnaire lubrique dans le ballet révolutionnaire offert par Chiang Ch'ing à ses hôtes 
américains; et lors de sa dernière intervention à l'acte III, il se borne à demander à Chou En-lai où se trouvent les toilettes (61). Le livret met tout particulièrement en évidence sa perplexité face aux jeux grammaticaux auxquels, dans le même temps, Mao se livre en virtuose. "You've got my vote. / I back the man who's on the right", lance le dirigeant communiste à Nixon à propos des élections prochaines. Kissinger tente aussitôt de corriger cette déclaration surprenante en modifiant la préposition : «Who's in the right you mean ", ce qui lui vaut un démenti sans équivoque : « No, no. / [...] I like right-wingers: Nixon, Heath...» (23). Le diplomate fait ainsi les frais du jeu sur la polysémie du terme "right» par lequel Mao indique qu'il soutient la «droite» américaine pour des raisons stratégiques même s'il ne lui donne pas « raison » sur le plan idéologique : l'un peut aller sans l'autre, contrairement à ce que semble penser Kissinger non sans fatuité. Si ce dernier représente la philosophie, il est clair que Mao figure ici la poésie, autrement dit une écoute du signifiant avant tout attentive à la manière dont, simultanément, il construit et interroge le sens des énoncés même les plus simples. Toutes considérations politiques mises à part, il n'est donc guère surprenant que le texte poétique de Goodman prenne si clairement parti contre l'homme d'État américain, enclin à négliger, au nom de la logique, les effets pragmatiques du discours.

\section{Logocentrisme et dramaturgie du livre}

19 Toutefois, l'analogie entre Nixon in China et le propos de McLuhan reste d'une portée limitée, car si Adams et Goodman comptent, via Cage, parmi ses héritiers intellectuels, le matériau grammatical qu'ils manipulent s'avère d'une richesse suffisante pour pousser sa réflexion dans ses derniers retranchements, voire pour faire apparaître quelques-uns des impensés qu'elle véhicule. Encore très marqué, à son corps défendant, par un héritage philosophique qu'il ne remet que partiellement en question, McLuhan cherche entre autres choses à rendre compte de l'emprise exercée par l'écrit dans tous les domaines de l'expérience humaine; or si la pensée elle-même est tributaire de l'écriture, alors peut-être faut-il en déduire que celle-ci est, à strictement parler, impensable, à moins bien sûr de revoir radicalement - plus radicalement peut-être que la philosophie n'est capable de le faire - ce que « penser » signifie.

Pour plusieurs raisons - notamment l'accent mis sur l'expérience sensorielle, sur le rôle de l'idéogramme comme Gestalt ou encore sur les effets d'intentionalité inhérents à la manière dont les figures construites par la technologie prennent sens par contraste avec un arrière-plan ("ground») dont elles sont inséparables - la réflexion de McLuhan s'inscrit dans le droit fil de la phénoménologie. Or il n'évite pas le biais logocentrique qui caractérise souvent ce type d'analyse, puisqu'il affirme sa croyance en l'unité primordiale d'un Verbe que l'adoption par les Grecs de l'alphabet phonétique aurait ensuite fragmenté de manière « catastrophique » (McLuhan, 1989, 32). Tout sauf postmoderne au sens de Lyotard, McLuhan élabore peu à peu un " grand récit », pour ne pas dire un mythe, destiné à mettre en lumière l'histoire tragique de la subjectivité occidentale; et ce récit s'appuie sur une référence de plus en plus insistante à la physiologie du cerveau humain, autrement dit sur une ontologie de la pensée que complète l'attention portée au pouvoir germinatif du logos, «seeds embedded in things animate and inanimate which structur $[e]$ and infor $[m]$ them and provid[e] the formal principles for their being and growth $»(33)^{4}$. 
21 Avant tout préoccupés par les questions liées au chant et à la composition musicales, Adams et Goodman auraient pu trouver des raisons de partager ce logocentrisme, qui est aussi un phonocentrisme puisque McLuhan affirme que l'écriture alphabétique, sous couvert de transcription phonétique, trahit l'expérience de l'oralité. Or c'est le contraire qui se produit : à tous les niveaux, l'écrit se montre prioritaire dans Nixon in China. Le livret de Goodman est l'œuvre d'une lettrée, voire d'une érudite ; son travail de composition poétique relève d'abord de la réécriture, et il suffit de lire On China, l'ouvrage semi-autobiographique où Henry Kissinger résume les enseignements qu'il a tirés de sa longue fréquentation de la Chine, pour mesurer avec quelle précision la scène de la rencontre entre Nixon et Mao (I.2) restitue la teneur des documents d'archives (Kissinger, 255-262). Qui plus est, le texte ne cesse de souligner que le discours des personnages puise constamment à des sources écrites. Mao reçoit ses visiteurs dans sa bibliothèque; à peine engagée, la conversation donne lieu à un compliment sur ses ouvrages (Kissinger : "The Chair- / Man may be gratified to hear / He's read at Harvard. I assign / All four volumes"; Goodman, 1987a, 21), politesse que le président chinois prend soin de rendre avant de prendre congé : «Six Crises isn't a bad book», observe Mao non sans condescendance, sur quoi Nixon souffle à Chou En-lai : "He reads too much" (30). De bout en bout, le livret s'avère truffé d'allusions intertextuelles parfois éclairantes, souvent délibérément incongrues, comme lorsque Mao emploie l'expression « Piecing the broken Golden Bowl » (27), traditionnelle dans la poésie chinoise (Cantagrel, 71, note 14) mais d'abord associée, dans l'esprit du spectateur anglophone, à l'Ecclésiaste (12:6) et au roman de Henry James. Le spectateur finit par en conclure que les personnages de Nixon in China ne peuvent prendre la parole sans mobiliser une vaste culture littéraire, que les énoncés qu'ils prononcent ne font sens que sur fond de lectures préalables et que le destin de la parole est, dans cet opéra, de revenir à l'écrit aussitôt qu'elle a été prononcée. C'est en tout cas ce que suggère la présence, aux côtés de Mao, de trois sténographes chargées de saisir au vol ses moindres propos et de les retranscrire pour la postérité.

22 On a noté plus haut que le livret attire l'attention sur le rôle de la télévision dans l'Amérique des années soixante-dix ; il est d'autant plus intéressant de constater que la conversation entre chefs d'État y porte avant tout sur les terrifiants pouvoirs de l'écriture. "The Chairman's books enthralled / A nation, and have changed the world", observe Nixon; à quoi Mao répond que l'écrit ne vaut qu'une fois devenu le guide de la pensée : "Those books of mine / Aren't anything. Incorporate / Their words into a people's thought / [...], then say / They live » (21). La véritable signification de cet échange en apparence badin apparaît à la fin de l'acte II lorsque Chiang Ch'ing, en proie à une inquiétante exaltation, brandit le Petit Livre rouge :

I am the wife of Mao Tse-Tung

Who raised the weak above the strong

When I appear the people hang

Upon my words, and for his sake [...]

I speak according to the book [...].

(57)

Comparable par sa fureur virtuose au second air de la Reine de la Nuit, cet impressionnant numéro de colorature dramatique semble de prime abord installer l'auditeur dans un univers logocentrique, puisqu'il ne laisse planer aucun doute quant aux désastres que peut entraîner le moindre mot prononcé, au nom du vieux président, par l'égérie de la Bande des Quatre. Pourtant, la suite du texte montre bien que la 
parole de Chiang Ch'ing doit sa dangereuse efficace à l'autorité de l'écrit. Le jeu de mots qui dit l'horreur de cette dérive totalitaire se présente d'abord comme un effet d'écriture : c'est le choix de placer le vocable « hang » à la fin d'un vers, juste avant un passage à la ligne, qui incite à y voir une allusion à la pendaison au lieu de le traiter comme un élément dé-sémantisé d'une expression toute faite. Qui plus est, le compositeur préserve ce double sens en ménageant une pause dans la mélodie après le premier énoncé du mot « hang » : en l'occurrence, c'est donc la lettre qui guide le chant. Point culminant et conclusion du deuxième acte, l'air de Chiang Ch'ing se termine fortissimo sur le mot «book» chanté douze fois sur un intervalle tendu de quinte augmentée dans le suraigu de la voix ; ainsi, le spectateur ne peut ignorer que Nixon in China relève, à tous les niveaux, d'une dramaturgie du livre ${ }^{5}$.

Si l'on se laisse guider par les grandes lignes de l'argumentation développée par McLuhan, on est tenté de conclure que Nixon in China attire ironiquement l'attention sur la façon dont ces acteurs majeurs de la vie politique internationale à l'ère de la mondialisation trahissent leur attachement nostalgique à une conception surannée de l'écrit, celle qui avait encore cours du temps de leur jeunesse, à l'époque de la Longue Marche et de la bataille du Pacifique (Goodman, 1987a, 61-70) ${ }^{6}$; mais une lecture fine de la partition et du livret laisse apparaitre une situation plus complexe. Dans The Global Village, McLuhan avance que tout nouveau média agit sur son environnement culturel de quatre manières que résume la figure de la « tétrade » $:$ il crée un nouveau mode de rapport au monde, il en frappe un autre d'obsolescence, il remet à l'honneur ( «retrieves ») un média précédemment devenu obsolète et il tend in fine à se confondre avec son propre contraire sous l'effet du passage à la limite que son succès même ne peut manquer d'entraîner (McLuhan, 1989, 10). Ce que l'opéra crée ou met en avant (" enhances »), c'est, on l'a dit, une alliance originale de l'œil, de l'oreille et de la main, une expérience holistique de la musique, de l'action scénique et du chant dans un espace où les images et les sons rayonnent de manière multi-directionnelle, faisant appel à l'intuition et à l'affect non moins qu'à la mémoire historique et à la réflexion politique. Du même coup, le théâtre d'Adams et de Goodman condamne la télévision à l'obsolescence : il s'aventure sur le même terrain qu'elle, mais pour aller beaucoup plus loin. Comme l'observe Michael Steinberg, cet opéra s'avère infiniment plus convaincant, plus "vrai », que les images d'archives même les plus fiables (Steinberg, 110). Lorsqu'il assiste à la rencontre entre Nixon et Mao, le spectateur a l'impression troublante de savoir enfin ce qui s'est réellement passé ce jour-là (116), non parce que le texte révèle des informations inédites - au contraire, il se borne à restituer sous forme versifiée ce que l'on savait déjà depuis longtemps - mais parce que le média choisi par Adams et Goodman est beaucoup plus « chaud » que la télévision, selon la terminologie de McLuhan : il fait preuve d'une " définition » supérieure qui favorise des effets de surdétermination, et il en appelle beaucoup moins à l'imagination du spectateur/auditeur, encouragé à rester passif face à un message saturé (McLuhan, 1964, 24-25) ${ }^{7}$. Du même coup, l'opéra remet à l'honneur la culture pré-télévisuelle de l'écrit, ce qui lui permet de redécouvrir le pouvoir mystérieux, à la fois créateur et destructeur, du livre. Tout sauf passéiste, l'art d'Adams et de Goodman ne cherche pas à interpréter coûte que coûte le nouveau à l'aune de l'ancien; au contraire, il s'aventure dans une direction imprévue, car si la présentation matérielle du livre (ce que McLuhan appelle la «figure ») n'a pas changé, l'arrière-plan ("ground») qui lui donne sens est tout à fait nouveau, ce qui en renouvelle profondément la fonction. 


\section{Écriture et expérience}

L'enjeu consiste donc à comprendre comment et à penser ce nouvel avatar de l'écriture sans s'appuyer sur un savoir acquis que tout incite à suspecter. Curieusement (ou peutêtre tout à fait naturellement, en raison de ses présupposés logocentriques), la grammaire que McLuhan appelle de ses vœux n'a rien d'une grammatologie ; jamais il ne s'engage dans une réflexion fine sur les spécificités des systèmes d'écriture, des "idéogrammes" orientaux - un linguiste parlerait plutôt de "logogrammes", de signes renvoyant à des mots entiers plutôt qu'à des «idées " appréhendées sans autre forme de médiation - ou de l'alphabet hâtivement qualifié de "phonétique» (formulation imprudente, car il revient à l'usage de déterminer le degré de proximité entre graphie et phonie non moins qu'à la forme ou à la répartition des lettres). À ce niveau, le propos de McLuhan n'est guère innovant, et il se contente de reprendre sans états d'âme quelques idées reçues, excepté dans les cas où elles posent manifestement problème. Or le livret de Nixon in China conteste d'emblée tous ces clichés, à commencer par la dichotomie, idéologiquement suspecte mais omniprésente chez McLuhan, qui oppose l'« Orient » à l'« Occident », même définis a minima comme de simples aires linguistiques.

26 Compte tenu du sujet traité, le spectateur serait par exemple en droit de s'attendre à ce que soit abordée la problématique de la traduction; or il n'y a pas dans Nixon in China un seul emprunt à la langue chinoise, excepté un «Gam bei ${ }^{8}$ " stéréotypé dans la scène du banquet (Goodman, 1987a, 35). Avec beaucoup de subtilité, Adams et Goodman tirent profit d'une convention du théâtre lyrique en vertu de laquelle tous les personnages parlent la langue du public, quelle que soit leur nationalité : Mao, Chou En-lai et Chiang Ch'ing s'expriment de bout en bout dans un anglais impeccable, ce que souligne ironiquement la présence de sténographes-interprètes auprès du président chinois (comme il n'y a rien à traduire, elles se contentent de répéter ses propos verbatim, ce qui produit un effet comique tout en accentuant le caractère non-directionnel d'un discours et d'un chant qui semblent le fait de plusieurs individus à la fois). Ainsi, la même langue - l'anglais standard - vaut tantôt pour le mandarin, tantôt pour l'idiome américain de Kissinger et de Nixon, ce qui la rend étrangement indéfinissable. Il ne s'agit pas de laisser entendre que le chinois et l'anglais sont en quelque sorte identiques; au contraire, l'attention du spectateur est attirée sur les obstacles qui empêchent la parole de circuler librement par la structure dramaturgique particulière de l'acte III, qui met en présence les deux couples présidentiels, le Secrétaire d'État américain et le premier ministre chinois mais évite avec soin de mêler leurs voix dans un grand sextuor à la manière de Lucia di Lammermoor 9 . Ce qui prédomine, c'est l'impression de ne plus savoir clairement où passe la frontière entre les différents idiomes: l'épouse du président Mao lance un retentissant «We'll teach / These motherfuckers how to dance! » avant d'exécuter avec son mari une langoureuse danse de salon (61), alors que jamais les personnages américains ne s'autorisent de telles expressions argotiques. A fortiori, il devient impossible de déterminer le statut exact des différents systèmes d'écriture à partir du moment où l'anglais le plus familier tient lieu d'une langue asiatique que des auteurs tels que Pound ou McLuhan considèrent, en raison de son recours à l'idéogramme, comme l'expression d'un rapport au monde radicalement étranger à l'épistémè occidentale. Euvre d'un poète indifférent à la reconnaissance sociale mais proche des milieux littéraires les plus innovants ${ }^{10}$ et d'un 
musicien lecteur de Walt Whitman, de Muriel Rukeyser et d'Emily Dickinson ${ }^{11}$, Nixon in China est un opéra au plus haut point "écrit ", c'est-à-dire moderniste au sens où l'entend Charles Bernstein. Pourtant, on ne peut en déduire qu'il insiste sur le rôle de la littéralité, car il est hasardeux de parler de « lettre » dans un contexte où l'alphabet est mis en position de fonctionner comme une écriture d'un type tout à fait différent sans pour autant perdre aucune de ses caractéristiques formelles.

Tout ceci entraîne des conséquences importantes quant aux rapports entre littérature et philosophie. À son corps défendant, McLuhan persiste à proposer une philosophie de l'écriture, c'est-à-dire à l'aborder à l'aune d'une idéalité abstraite considérée indépendamment de ses déclinaisons empiriques. Cette idéalité, c'est ce que désigne en anglais le mot "word", autrement dit le Verbe (le logos) présenté comme une réalité accessible aux sens, mais implicitement opposé aux manifestations visibles ou audibles du langage (Understanding Media comporte trois chapitres respectivement intitulés The Spoken Word, The Written Word et The Printed Word: dans ces conditions, on est en droit de se demander à quoi le mot "word" renvoie si, pris isolément, il ne désigne précisément ni la parole, ni l'écrit, ni le texte imprimé). Quoique concret (puisqu'il se prête à une étude phénoménologique), le Verbe selon McLuhan ne peut être saisi sous tous ses aspects lors d'aucune expérience particulière et ne se manifeste jamais que de manière fragmentaire - d'où l'allusion qu'il fait, dans Understanding Media, à la Tour de Babel :

Language [...] may have been the "Tower of Babel" by which men sought to scale the highest heavens. Today computers hold out the promise of a means of instant translation of any code or language into any other code or language. The computer, in short, promises by technology a Pentecostal condition of universal understanding and unity [...] [a] condition of speechlessness that could confer a perpetuity of collective harmony and peace.

(McLuhan, 1964, 87).

Ouvertement marqué par l'héritage chrétien, ce discours millénariste tranche curieusement sur le texte de Goodman, croyante et prête à glisser des citations bibliques dans Nixon in China mais, sur le fond, beaucoup moins disposée à s'aventurer en direction d'une théologie de l'écriture. Prononcés par Chou En-lai, les derniers mots du livret font écho au Nouveau Testament: "Outside this room the chill of Grace / Lies heavy on the morning grass " (Goodman, 1987a, 71). Incongrue dans la bouche d'un personnage historique connu pour son adhésion à un communisme officiellement athée, cette allusion aux Actes des Apôtres (2:1-4) suscite avant tout la perplexité, d'autant que le texte fait référence à un concept chrétien alors que son auteur se revendiquait encore, à l'époque de la rédaction, du judaïsme réformé ${ }^{12}$. L'image paradoxale sur laquelle se clôt cet opéra est celle d'une Pentecôte à l'envers : Chou n'a rien d'un disciple de Jésus, la grâce divine agit à l'extérieur plutôt que dans la chambre où il s'est retiré, et la métaphore qui sert à l'évoquer est celle du givre alors que le Nouveau Testament décrit les « langues de feu » envoyées par l'Esprit Saint (Actes $2: 3$ ). Qui plus est, on note qu'à l'exception problématique du terme "grace», Goodman ne manie pas le lexique de la transcendance, mais celui de la sensation: Chou évoque une impression de froideur, de pesanteur, l'effet particulier que produit le chant des oiseaux lorsqu'il s'élève juste avant l'aurore ("Just before dawn the birds begin, / The warblers who prefer the dark, / The cage-birds answering »; 71) ${ }^{13}$. Le propos relève ainsi d'une approche empiriste pour laquelle la grâce n'existe que sur le mode de 
l'appréhension sensible, de telle sorte que son rapport avec le logos (notamment, mais pas seulement, celui de la théo-logie) reste entièrement à définir, si tant est qu'il existe.

Si le texte de Goodman met ainsi en avant le contact empirique avec le monde - voire, au premier chef, avec le langage et l'écriture - sans plus tenir pour acquise la possibilité d'accéder ainsi à l'idéalité, alors c'est le concept même d'expérience que Nixon in China invite à repenser, ce qui entraîne des répercussions considérables. La tradition empiriste classique et sa critique kantienne ou post-kantienne s'accordent à voir en elle la première étape du processus qui permet l'élaboration d'un savoir abstrait. Mise à l'honneur par la phénoménologie, cette idée se présente comme la reformulation moderne d'une intuition beaucoup plus ancienne, celle qui distingue pour les articuler le monde sensible et la sphère de l'intelligible. Ainsi se pose la question de la manière dont peut être pensé l'écrit dès lors qu'il s'appréhende comme une simple marque empirique sans rapport nécessaire avec quoi que ce soit qui relève de la " dialectique ", en rupture radicale avec la tradition logocentrique ${ }^{14}$. L'enjeu pour la philosophie est immense. Derrida a formulé une critique du logocentrisme au nom de l'écriture, sur la base d'une grammatologie infiniment moins sommaire que celle de McLuhan; mais il refuse avec fermeté d'assimiler l'écrit à ce qui, en lui, s'offre à l'expérience sensible, notion trop liée aux métaphysiques de la présence pour ne pas restaurer d'une autre manière la "transcendantalité " dont il entend se défaire (Derrida, 90). Lorsqu'il en appelle à une nouvelle pensée du langage capable de passer à la fois par l'audition, le regard et le toucher sans se sentir tenue d'y discerner, à l'instar de McLuhan, la mystérieuse présence d'un logos analogue à un Dieu caché dans l'attente de la révélation apocalyptique, l'opéra d'Adams et de Goodman ne peut donc s'engager que dans une seule direction, celle d'un examen radical de tout ce que l'on croit savoir à propos de l'expérience. C'est peut-être là ce qui justifie en dernière analyse la référence à la Chine, car si la philosophie occidentale n'a pour l'heure guère de réponses à apporter à un tel questionnement, la tradition taoïste chère à John Cage pourrait bien fournir quelques précieux éclaircissements sur ce point, si l'on en croit les analyses de François Jullien.

Ne s'exprimant pas dans le langage de l'Être, la pensée chinoise est au contraire à l'aise pour prêter attention au stade [...] où le sensible se résorbe et se déspécifie, se disqualifie, "s'affadit", sans pour autant verser dans l'invisible de la métaphysique ; où les démarcations se défont et qui, dans son indifférenciation, laisse apparaître l'incessante transition des choses.

(Jullien, 237)

\section{BIBLIOGRAPHIE}

«Up Front : John Adams », entretien avec John Adams, New York Times, 19 novembre 2010, en ligne, http://www.nytimes.com/2010/11/21/books/review/Upfront-t.html?ref=review\&_r=0 (page consultée le 12 mars 2013). 
ADAMS, John, « The Zen of Silence », New York Times, 19 novembre 2010, en ligne, http:// www.nytimes.com/2010/11/21/books/review/Adams-t.html?ref=review (page consultée le 12 mars 2013).

---, et Alice GOODMAN, Nixon in China, interprété par Sanford Sylvan, James Maddalena, Thomas Hammons, John Duykers, Carolann Page et Trudy Ellen Craney, Orchestra of St. Luke's dirigé par Edo de Waart, Nonesuch Records 7559-79177-2 [double CD], 1988 (1987).

ALFANDARY, Isabelle, Le Risque de la lettre : lectures de la poésie moderniste américaine, Lyon, ENS Éditions, 2012.

ANGELOFF, Tania, Histoire de la société chinoise 1949-2009, Paris, La Découverte, 2010.

BÉGHAIN, Véronique, Les Aventures de Mao en Amérique, Paris, Presses Universitaires de France, 2008.

BERNSTEIN, Charles, A Poetics, Cambridge, MA, Harvard University Press, 1992.

CAGE, John, « Composition as Process », Silence: Lectures and Writings, Londres, Marion Boyars, 1968 (1958), 18-56.

CANTAGREL, Laurent, Nixon in China d'Alice Goodman, traduction et annotations, L'Avant-scène opéra 267, 2012, 13-72.

DERRIDA, Jacques, De la Grammatologie, Paris, Minuit, 1967.

GOODMAN, Alice, Nixon in China, livret (1987a), L'Avant-scène opéra 267, 2012, $13-71$.

---, « Towards Nixon in China », essai joint au livret de Nixon in China, Nonesuch Records 7559-79177-2 [double CD], 1988 (1987b), 11-13.

JAYNES, Julian, The Origin of Consciousness in the Breakdown of the Bicameral Mind, Boston, Houghton Mifflin, 2000 (1976).

JULLIEN, François, Les Transformations silencieuses, Paris, Grasset \& Fasquelle, 2009.

KAMUF, Peggy, " The Replay's the Thing », dans David J. Levin (dir.), Opera Through Other Eyes, Stanford, CA, Stanford University Press, 1993, 79-105.

KISSINGER, Henry, On China, Londres, Penguin Books, 2011.

LAOZI, Daodejing, traduit du chinois par Edmund Ryden, Oxford, Oxford University Press, 2008.

LYOTARD, Jean-François, La Condition postmoderne, Paris, Éditions de Minuit, 1979.

MARX, Karl, «Ad Feuerbach (Thèses sur Feuerbach) », traduit de l'allemand par Maximilien Rubel avec la collaboration de Louis Évrard et Louis Janover, dans Karl Marx, Philosophie, Paris, Gallimard, 1982 (1845), 232-35.

McLUHAN, Marshall, Understanding Media: The Extensions of Man, Abingdon, Routledge, 1964.

---, et Bruce R. POWERS, The Global Village: Transformations in World Life and Media in the $21^{\text {st }}$ Century, New York, Oxford University Press, 1989.

PERLOFF, Marjorie, The Dance of the Intellect: Studies in the Poetry of the Pound Tradition, Evanston, IL, Northwestern University Press, 1996 (1985).

POUND, Ezra, ABC of Reading, New York, New Directions, 2010 (1934).

RANCIÈRE, Jacques, Le Partage du sensible, Paris, Galilée, 2000.

RIGAUDIERE, Pierre, «Introduction et guide d'écoute », L'Avant-scène opéra 267, 2012, 8-72. 
ROSS, Alex, «The Harmonist: John Adams Takes the Agony out of Modern Music », The New Yorker, 8 janvier 2001, en ligne, http://www.earbox.com/inter011.html (page consultée le 12 mars 2013).

STEINBERG, Michael, «Nixon in China », dans Thomas May, dir., The John Adams Reader: Essential Writings on an American Composer, Pompton Plains, NJ, Amadeus Press, 2006 (1988), 110-19.

\section{NOTES}

1. Mort en septembre 1976, Mao a eu pour successeurs Hua Guofeng, puis, à partir de 1978, Deng Xiaoping ; celui-ci mène tout au long des années quatre-vingt et quatre-vingt-dix une politique de démaoïsation que résume le slogan des «Quatre Modernisations » (en matière d'agriculture, d'industrie, de développement technologique et de défense nationale) (Angeloff, 51). En l'absence de toute libéralisation politique, les effets inégalement répartis de la croissance économique ont commencé à déstabiliser la société chinoise, prélude à une crise de régime qui se traduira en maijuin 1989, deux ans après la création de Nixon in China, par les événements de la place Tiananmen (66). Tombé en disgrâce à partir de 1975 et partisan déclaré des réformes, Zhou Enlai (Chou Enlai) est mort en février 1976. Jiang Qing (Chiang Ch'ing) a été condamnée à mort en janvier 1981 pour son rôle au sein de la Bande des Quatre; en 1983, sa peine a été commuée en prison à vie (elle mourra en 1991). Définitivement discrédité par le scandale du Watergate qui a éclaté en juin 1972, Richard Nixon a démissionné en août 1974; il a eu pour successeurs Gerald Ford (1974-1977), Jimmy Carter (1977-1981), puis Ronald Reagan qui, en 1987, arrive au terme de son second mandat. Maintenu par Gerald Ford dans ses fonctions de Secrétaire d'État, Henry Kissinger s'est retiré de la politique en 1977, après la victoire de Carter ; enseignant à l'Université de Georgetown, il dirige depuis 1982 un cabinet de conseil.

2. Dans le livret de Nixon in China, les noms propres d'origine chinoise sont transcrits selon le système phonétique Wade-Giles encore en usage dans les pays de langue anglaise. En pinyin, le système de romanisation recommandé par les autorités chinoises, ce nom s'orthographie «Zhou Enlai ».

3. Dans la production de Chen Shi-Zheng, donnée au Théâtre du Châtelet en avril 2012, la scène était surmontée d'une grappe de moniteurs vidéo où étaient projetés des films d'actualité contemporains des événements. Paradoxalement, les images "authentiques", réellement retransmises en direct, se trouvaient ainsi appartenir à un « hier » bien plus lointain que celui auquel se réfère, dans le livret, le personnage semi-fictif de Nixon.

4. À la fin de sa vie, McLuhan s'intéresse de plus en plus à la latéralisation du cerveau, dont l'hémisphère gauche privilégierait la pensée abstraite et la vision analytique au détriment des modes d'appréhension intuitifs et haptiques que McLuhan associe à l'hémisphère droit. Sur ce point, sa réflexion fait écho à celle du psychologue Julian Jaynes qui explique l'émergence de la conscience par l'apparition de nouveaux modes de fonctionnement neuronal à la fin de l'Âge du Bronze, en réaction à de profonds bouleversements sociaux. Marquée par l'héritage de William James, de Wittgenstein et de la philosophie analytique, l'anthropologie spéculative de Jaynes prend le relais de la réflexion métaphysique sur le dualisme corps-esprit («the mind-body problem»; Jaynes, 3) pour suggérer que la conscience est avant tout une métaphore: construction langagière liée à l'émergence de l'écriture, elle serait parvenue à supplanter les modes ancestraux de réceptivité aux hallucinations auditives que génère l'hémisphère droit du cerveau et que l'humanité préhistorique aurait crues d'origine surnaturelle. Ainsi, la psychologie jaynesienne se présente comme une onto-théologie au sens strict du terme puisqu'elle affirme l'existence des dieux, assimilés au logos primitif tel qu'il se déployait avant l'invention de l'écriture. 
5. Cela vaut aussi sur le plan scénique : si le geste impérieux par lequel Chiang Ch'ing brandit le Petit Livre rouge marque la fin de l'acte II, la deuxième scène de l'acte I se déroule quant à elle dans la bibliothèque où eut lieu l'unique rencontre des présidents américain et chinois. Véronique Béghain y voit une manière de situer le maoïsme dans la tradition littéraire chinoise, mais aussi d'attirer l'attention sur l'ambivalence des rapports qu'entretenait Mao avec l'écrit, associé à l'idéalisme bourgeois et au rôle néfaste des lettrés dans la Chine impériale (116-120).

6. Peggy Kamuf rappelle que Nixon fit les frais de l'intrusion des médias modernes dans le jeu politique lorsqu'il sortit perdant des premiers débats télévisés entre candidats à la MaisonBlanche pendant la campagne présidentielle de 1960 : témoin et acteur des mutations culturelles qu'entraîna le recours de plus en plus fréquent à la caméra, il avait de bonnes raisons de regretter l'époque où l'image ne concurrençait pas encore la presse écrite (86).

7. Encore employée dans les pays de langue anglaise pour qualifier ce type d'ouvrage, l'expression de «CNN opera » s'avère quelque peu ambiguë. Si elle suggère qu'une œuvre telle que Nixon in China aborde un sujet de nature à flatter les goûts du public habitué à regarder les chaînes d'information continue, elle sous-entend aussi que ce type de création lyrique se donne pour matériau la télévision en tant que telle, qu'il a pour objet d'en retrouver les procédés et les effets grâce à des emprunts directs, ce qui n'est pas du tout le cas, comme on vient de le voir. Par ailleurs, l'expression de « $\mathrm{CNN}$ opera » paraît déjà datée à l'heure de la télévision sur Internet et des actualités diffusées en temps réel sur les tablettes tactiles; elle présente ainsi l'intérêt de souligner l'historicité de ce type de théâtre, sans doute moins liée à son sujet (au "message » qu'il délivre à propos des relations sino-américaines) qu'à sa place dans l'évolution technologique, bien différente - en dépit de quelques similitudes formelles - de celle qu'ont pu occuper en leur temps les opéras historiques de Meyerbeer ou de Verdi.

8. «À votre santé !»

9. « [L]'on n'a jamais ici cinq voix simultanément présentes, mais généralement trois, avec deux très brefs passages à quatre voix. Se pose la question de savoir si cette absence de quintette réel est liée à une réticence d'ordre dramaturgique, [...] ou si John Adams n'a pas voulu affronter l'écriture à cinq voix réelles", s'interroge Pierre Rigaudière (58). Outre qu'il y a bien six personnages sur scène et non pas cinq au début de l'acte III - même si Kissinger ne fait que quelques interventions discrètes avant de s'absenter définitivement - on peut trouver surprenant que John Adams se voie soupçonné de reculer devant les exigences techniques d'une polyphonie complexe, lui qu'un tel défi n'effraie pas dans d'autres partitions (ainsi, la scène de la transformation dans A Flowering Tree [2006] prend manifestement exemple sur Lux ceterna [1966] de György Ligeti, célèbre chœur à seize voix réelles). Il y aurait sans doute beaucoup à dire sur la manière dont une partie de la critique musicale française s'obstine à instruire le procès en incompétence de ce compositeur américain formé à Harvard.

10. Aujourd'hui pasteur au Trinity College de Cambridge, Alice Goodman est l'épouse du poète britannique Geoffrey Hill.

11. Le livret de Harmonium (1981), cantate pour chœur et orchestre, rassemble des poèmes de John Donne et d'Emily Dickinson, tandis que le texte de The Wound-Dresser, pour baryton et orchestre, est emprunté à Whitman. Dans Doctor Atomic (2005), le principal personnage féminin chante plusieurs airs composés d'après des poèmes de Rukeyser. Le livret de El Niño (2000) est construit à la manière d'une anthologie poétique, tout comme celui de The Gospel According to the Other Mary (2012).

12. La conversion de Goodman à l'anglicanisme est intervenue au début des années quatre-vingtdix alors qu'elle travaillait au livret de The Death of Klinghoffer (1991), son second opéra.

13. Allusion à John Cage qui, avec humour, avait suggéré d'intituler «For the Birds » son livre d'entretiens avec Daniel Charles?

14. Quoique d'inspiration derridienne, l'intéressant article de Peggy Kamuf n'aborde pas cette problématique; il étudie la «dissémination » de la présence sous l'action de médias que Kamuf 
analyse en des termes empruntés à Walter Benjamin (85). Quant à Véronique Béghain, elle consacre de belles pages au rôle symbolique du livre dans Nixon in China (111-125), mais sans articuler clairement les concepts de «texte », de "théorie » et de "littérature » (125). Or non seulement l'enjeu consiste à penser l'écriture sans recourir aux catégories héritées de l'idéalisme (au nombre desquelles figure l'opposition entre "théorie» et "pratique»), mais c'est précisément le travail littéraire de Goodman qui attire l'attention sur les propriétés infratextuelles de l'écrit, sur ce qui en lui est offert à l'expérience mais n'entre dans aucun rapport défini d'avance avec quoi que ce soit qui relève du logos. Béghain a certes raison de signaler que le rapport entre "théorie" (idéalité) et praxis révolutionnaire est un point névralgique de l'idéologie maoïste, comme le rappellent les propos du président chinois au début de l'acte II (113) ; à vrai dire, ce débat remonte à Marx lui-même, qui dans les Thèses sur Feuerbach réclame en philosophe une percée décisive hors de la philosophie, associée aux dérives réactionnaires de l'idéalisme bourgeois : «Les philosophes n'ont fait qu'interpréter le monde de diverses manières ; ce qui importe, c'est de le transformer » (Marx, 235). Toutefois, il n'y a là qu'un des aspects du problème tel qu'il est posé dans Nixon in China; et l'on pourrait dire de même que la librettiste et le compositeur tentent, en littéraires, une percée hors de la littérature à la faveur du « dialogue des genres" (Béghain, 77) propre à l'opéra lorsqu'ils délaissent les lettres au profit d'une réflexion sur la lettre qui est bien loin de ne concerner que la poésie dramatique. Enfin, on est tenté d'ajouter que cette double interrogation sur la possibilité d'un accès au logos ne peut manquer de revêtir une dimension religieuse, puisque cette question joue un rôle déterminant dans la tradition chrétienne depuis l'Évangile de Jean: croyante, Goodman s'est trouvée confrontée à quelque chose qui mettait en cause quelques-unes des formulations dogmatiques de la croyance. Cette triple percée (hors de la philosophie, de la littérature et du dogme religieux) contribue à expliquer la rencontre paradoxale du marxisme et du questionnement théologique qui s'opère à la fin de Nixon in China sous les auspices du théâtre lyrique.

\section{RÉSUMÉS}

Évocation de la rencontre historique entre l'Amérique des années soixante-dix et la Chine maoïste, Nixon in China de John Adams et Alice Goodman attire l'attention sur le rôle cognitif de l'écriture et notamment sur les différentes manières dont elle structure le rapport au monde selon qu'elle est alphabétique ou idéogrammatique. Récurrente tout au long du $20^{\mathrm{ème}}$ siècle nordaméricain, cette interrogation trouve sans doute sa formulation la plus développée dans les travaux de Marshall McLuhan; aussi est-ce d'abord vers ses travaux que l'on se tournera d'abord afin d'identifier la contribution singulière de Nixon in China à ce débat engagé de longue date. Jusqu'à un certain point, l'analyse confirmera le bien-fondé d'un tel rapprochement, mais elle en fera également percevoir les limites. Étudier Nixon in China à la lumière de Understanding Media, c'est aussi s'interroger sur le média nouveau qu'inventent Adams et ses collaborateurs, sur ses rapports avec les médias existants, voire sur la fonction inattendue qu'il leur attribue dans un contexte transformé par son apparition. Or la principale innovation de Nixon in China consiste de ce point de vue à élaborer une dramaturgie du livre dont ne saurait rendre compte la grammatologie trop sommaire que propose McLuhan. On ne saurait, du reste, lui en faire grief, car la pensée de l'écriture vers laquelle fait signe Nixon in China n'existe nulle part en philosophie, pas même chez Derrida qui pourtant s'interroge sur ses conditions de possibilité, mais pour aussitôt l'exclure de son champ d'étude. Le présent travail n'aura pas pour ambition d'élaborer 
cette grammatologie nouvelle, mais tout au plus de suggérer, sur le mode hypothétique, à quoi elle pourrait ressembler.

In Nixon in China, composer John Adams and librettist Alice Goodman recount 1970s America's historic encounter with Maoist China. In the process, they draw attention to the cognitive function of writing and especially to the different ways in which ideogrammatic and alphabetic writing systems shape experience and thought. While this question was repeatedly raised in North America throughout the twentieth century, its most detailed formulation occurs in the works of Marshall McLuhan; thus, his study of writing as a medium will be invoked in an attempt to highlight the opera's original contribution to this long-standing debate. Close analysis both confirms that such a parallel is justified up to a point and reveals its limitations. No comparison between Nixon in China and Understanding Media can be complete unless it involves an examination of the new medium invented by Adams and his collaborators, an analysis of its relationship with previously existing media, and a description of the new functions it assigns them. In this regard, Nixon in China mainly innovates by exploring the theatrical potential of the book in ways to which McLuhan's sketchy grammatology is unable to do justice. This points to a wider problem, as approaches to writing of the kind prefigured in Nixon in China are nowhere to be found in philosophy, not even in the works of Derrida, who wonders whether they could exist, yet immediately answers this question in the negative. The purpose of the present paper is not to invent such a grammatology but, less ambitiously, to formulate a few hypotheses about the shape it may eventually take.

\section{INDEX}

Keywords : Nixon in China, John Adams, Alice Goodman, Marshall McLuhan, Jacques Derrida, writing, alphabet, ideogram, logocentrism, experience

Mots-clés : Nixon in China, John Adams, Alice Goodman, Marshall McLuhan, Jacques Derrida, écriture, alphabet, idéogramme, logocentrisme, expérience

\section{AUTEUR}

\section{MATHIEU DUPLAY}

Université Paris Diderot - Paris 7 